\title{
INTRA-HOSPITAL TRANSPORTATION OF EXTREMELY PRETERM INFANTS AND INCIDENCE OF INTRAVENTRICULAR HAEMORRHAGES
}

N. Wadström ${ }^{1}$, M. Breindahl' ${ }^{1}$, B. Hallberg ${ }^{1,2}$, B. Skiöld ${ }^{1,3}$

${ }^{1}$ Patient Area Neonatology, Karolinska University Hospital, Stockholm, Sweden

${ }^{2}$ Dept CLINTEC and ${ }^{3}$ Dept of Women's and Children's Health, Karolinska Institutet, Stockholm, Sweden

Inter-hospital transportation of extremely preterm infants is a known risk factor for intraventricular haemorrhages (IVH) ${ }^{1,2,3}$. The morphology of the brain and its vessels is immature and autoregulation of the cerebral blood flow is poor, which in combination with vibrations and linear acceleration may result in harmful intracranial bleedings ${ }^{4}$

When the new Karolinska University Hospital opened in November 2016, the Neonatal- and Delivery units were separated, resulting in a 900-meter long intra-hospital culvert transfer of all admitted infants. Data on whether intra-hospital transportation is associated with IVH is scarse.

Aim To compare short-term mortality and incidence of IVH following intra-hospital transfer in extremely preterm infants during 24 months before and 22 month after the move.

Table 1. Perinatal characteristics of infants born Nov 27th 2016 to Oct 4th $2018(n=261)$

\begin{tabular}{|l|c|c|}
\hline & $\begin{array}{c}\text { Controls } \\
\mathbf{N = 1 4 9}\end{array}$ & $\begin{array}{c}\text { Transported } \\
\mathbf{n = 1 1 2}\end{array}$ \\
\hline $\begin{array}{l}\text { Gestational age: mean } \pm \text { SD } \\
\text { range (w+d) }\end{array}$ & $\begin{array}{c}24+4 \pm 1.28 \\
(21+3-26+6)\end{array}$ & $\begin{array}{c}24+5 \pm 1.1 \\
(22+3-26+6)\end{array}$ \\
\hline $\begin{array}{l}\text { Birth weight (BW): mean } \pm \text { SD } \\
\text { range (g) }\end{array}$ & $\begin{array}{c}659 \pm 147 \\
(275-1022)\end{array}$ & $\begin{array}{c}699 \pm 149^{*} \\
(370-1160)\end{array}$ \\
\hline Boys, $\mathrm{n}(\%)$ & $85 / 149(57)$ & $55 / 112(49)$ \\
\hline Multiple pregnancies, $\mathrm{n}(\%)$ & $32 / 149(22)$ & $30 / 112(27)$ \\
\hline $\begin{array}{l}\text { Normal, non-instrumental vaginal } \\
\text { delivery, } \mathrm{n}(\%)\end{array}$ & $60 / 149(40)$ & $53 / 112(47)$ \\
\hline Caesarian delivery, $\mathrm{n}(\%)$ & $89 / 149(60)$ & $59 / 112(53)$ \\
\hline APGAR <7 at 5 min, $\mathrm{n}(\%)$ & $85 / 147(58)$ & $55 / 111(50)$ \\
\hline
\end{tabular}

${ }^{*} p=0.03$ for BW, all other NS (not statistically different), $p>0.05$, Student's $t$-test/ Chi square

Methods All infants born $<27$ weeks +0 days during 22 months following the move ("transported group", $n=112$ ) were compared to a group of infants born during the 24 preceding months ("control group", $n=149$ ). Infants born after the move were subjected to an additional early cranial ultrasound prior transportation, most often before six hours' age. All other care was provided according to standard hospital protocols, and the remainder of the study was observational.

Ultrasound reports were reviewed by two neonatologists and graded according to a modified Papile classification ${ }^{5}$;

$$
\begin{aligned}
& \text { I: Subependymal/ germinal matrix haemorrhage } \\
& \text { II: Limited IVH without ventricular dilatation }(<50 \%) \\
& \text { III: Extensive IVH with ventricular dilatation }(>50 \%) \\
& \text { IV: Any grade IVH with accompanying parenchymal haemorrhagic infarction }
\end{aligned}
$$

Parents received oral and written information prior to delivery and written consent was obtained. Statistical analyses were performed using PASW Statistics 23.0 (SPSS Inc, Chicago, Illinois, USA), MedCalc Software bvba and Microsoft Excel.

The study was approved by the Ethical Review Board in Stockholm, (2016/2500-31)

Figure 1. Short term mortality, before and after the move (NS)

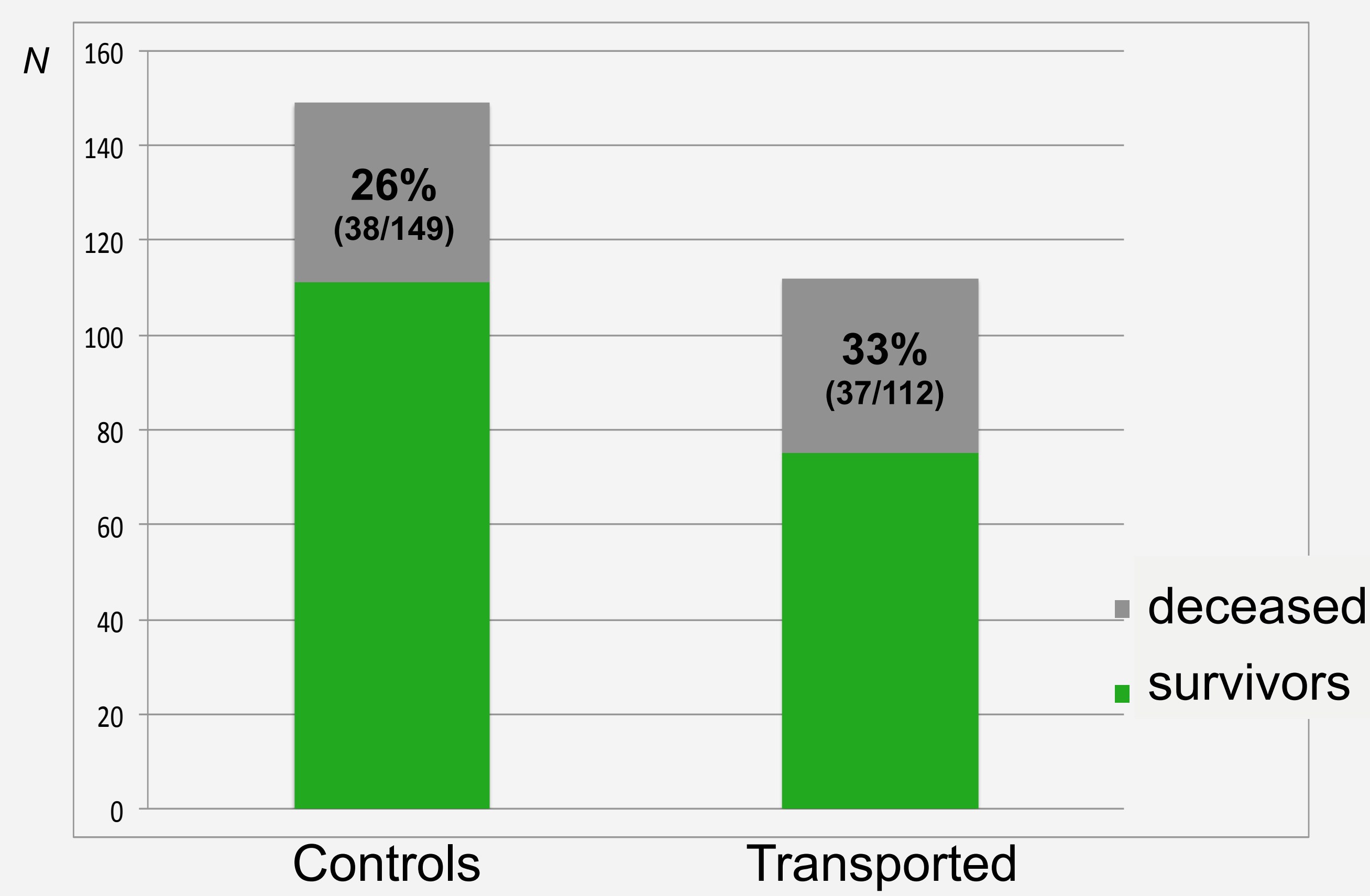

1. Hilary EA Whyte et al. Paediatr Child Health 2015

1. Mohamed MA, Aly H. et al. Arch Dis Child Fetal Neonatal Ed. 2010

3. Rennie \& Roberton's Textbook of Neonatology $5^{\text {th }}$ Ed. 2012

4. Blaxter L. et al. J Engineering Med 2017

5. Papile LA et al. J Pediatr 1978

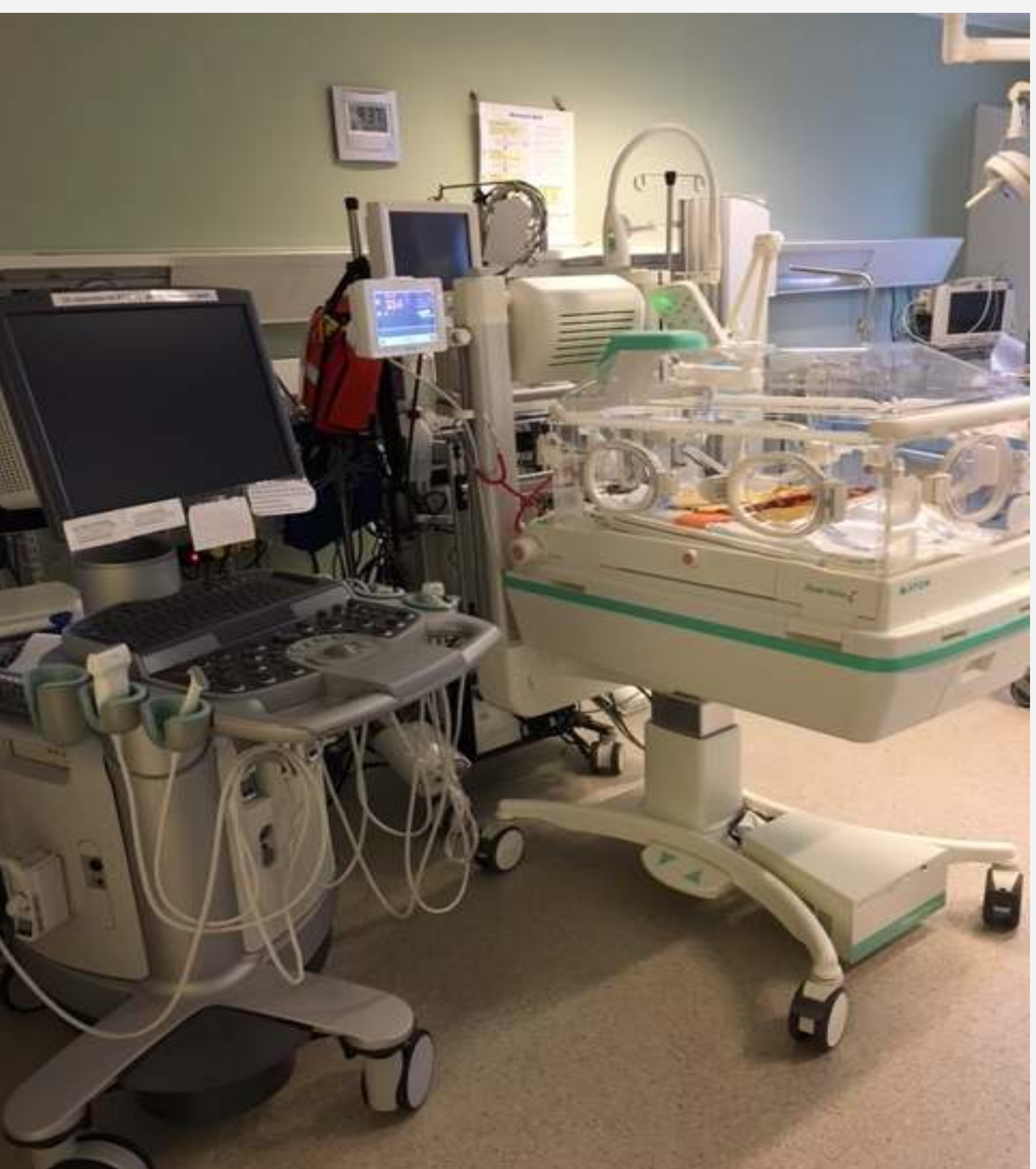

Results 261 extremely preterm infants were born during the study period; $n=112$ transported and $n=149$ controls. Infants were similar with regards to perinatal characteristics except for BW which was higher in the transported group (table 1).

Mortality was not different between groups; $33 \%$ (37/112) in transported vs $26 \%(38 / 149)$ in controls (fig 1$)$.

IVH: The incidence of any IVH was similar between groups: $37 \%$ $(39 / 106)$ in transported infants vs $47 \%(60 / 127)$ in controls (fig 2). We found no differences in low-grade or higher grade IVH or unior bilateral affection of lesions (data not shown). 28 infants died before ultrasound could be performed.

Figure 2. Incidence of IVH before and after the move (all NS)

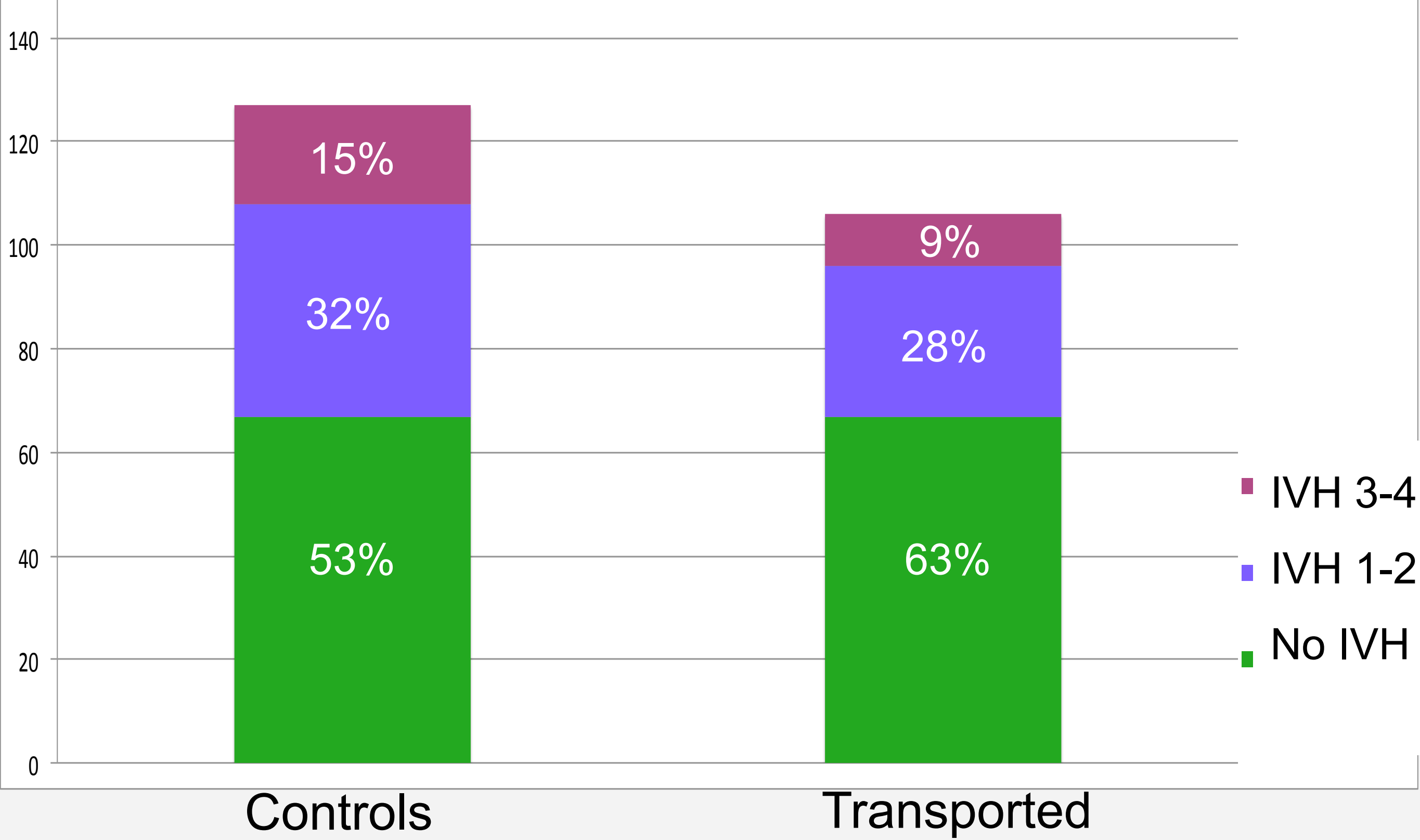

CONCLUSIONS

Our preliminary analysis of extremely preterm infants exposed to an early intra-hospital culvert transfer of $900 \mathrm{~m}$ revealed no significant differences in mortality or IVH. Data collection will continue until the delivery ward has moved into the new

hospital, and long-term outcomes will be determined before definitive conclusions on safety can be drawn. 